\title{
WIRELESS LOCAL AREA NETWORKS AND MOBILE DEVICES TO ACTUALIZE THE NOTION OF UBIQUITOUS COMPUTING IN LIVING CLASSROOMS
}

\author{
$A$ case study in teaching and learning Astrophysics
}

\author{
Serena Pastore \\ INAF - Astronomical Observatory of Padova, vicolo Osservatorio $5-35122-P A D O V A-$ \\ $I T A L Y$
}

Abstract: The integration of wireless local area network and wireless mobile devices allows to implement a complete information system able to support everyday activities unobtrusively and seamlessly as the ubiquitous computing paradigm says. Wireless mobile ad hoc networking could be successfully used in the construction of flexible and adaptive information system with no fixed infrastructure and it allows to covers multi-hop scenarios such as m-learning approaches. This paper describes choices and issues encountered in the adoption of wireless technologies as regards standards and topologies in order to realize a wireless network infrastructure suitable for teaching and learning Astrophysics using mobile devices. This study is part of the "Learning form Starlight" project presented by the Italian National Institute for Astrophysics to the Hewlett Packard Philanthropy foundation aiming at introducing in schools a new way of teaching and learning not strictly related to the classroom location context. The realization of this system outlines that the Wi-Fi standard in network deployment has up to now to be preferred to other available wireless technologies for the easiest way of set up connection and use and the possibility to implement different network topologies suitable for m-learning applications.

Key words: wireless local area networks (WLAN), ubiquitous computing, ad-hoc networks, 802.11 standards. 


\section{INTRODUCTION}

Ubiquitous computing [1] means that the computing system becomes a part of everyday environment, and user's interaction with it is available whenever he/she needs. The achievement of such vision necessarily requires an adequate wireless network infrastructure. Ubiquitous technology is characterized by the attributes of mobility, interconnectivity and contextawareness. Mobility could be realized using devices easily transportable and simple to interact with: challenges are limited graphics capabilities, minimal screen dimensions, memory, disks and battery duration. Interconnectivity should support not only point-to-point connection, but also additional capabilities such as being aware of each other or knowing how to exchange information. The concept of the context aware means the recognition of network, protocol, software, hardware (knows as the resource qualification) when interacting with other devices. A mobile learning ${ }^{1}$ environment is an appropriate scenario to put ubiquitous computing into practice since it allows to build a technology-supported classroom environment that is independent by the physical location. The major function of this virtual classroom is to capture and automatically integrate various information of teacher's lecture, including presentation-style lectures, notes, materials, voice and images to form available multimedia courseware so as to facilitate users' access and learning. By using mobile hardware such as handwriting tablets and palms with wireless connectivity, this interactive networked environment enables collaborative learning with groupware functions. The strict integration of mobile devices, wireless communication and network technology allows the utilization of computing power for teacher and students anytime and anywhere and the interconnection with other offering applications and services devices seamlessly.

With the vision to actualize ubiquitous computing in an educational environment, this paper describes the work and the technological solutions adopted in terms of wireless standards and network topologies to implement a wireless information system suitable to enhance teaching and learning Astrophysics in schools of different grades in Italian education. The document finally outlines the main issues encountered in network deployment.

\footnotetext{
${ }^{1}$ Mobile learning (m-learning) is the intersection of mobile computing and e-learning: it's a kind of e-learning independent of location, time or space.
} 


\section{BACKGROUND: THE "LEARNING FROM STARLIGHT" PROJECT}

The "Learning from Starlight project" was presented by the Italian National Institute for Astrophysics to the Hewlett Packard Philanthropy Foundation [2] with the aim of allowing Italian students to realize that Astrophysics is a science based on observation of signals (light and other electromagnetic waves). The overall goal is to demonstrate that mobile devices and wireless technology could improve learning and teaching. The project was selected by the foundation that granted a package consisting in a consistent number of handled devices such as Personal Digital Assistant PDAs (HP iPAQ Pocket PC) and notebooks (HP TabletPC) [3] all equipped with a Microsoft Windows ${ }^{2}$ environment and wireless adapters for Wi-Fi [4], Bluetooth [5] and infrared connections together with other devices useful for network support (access points, printers, digital projectors).

The project is included in the field of mobile learning [8] by enabling the use of portable computing devices over wireless networks. Mobile computing in education extends learning and teaching activities to spaces beyond the traditional classroom even if teachers and learners gain increased flexibility and new opportunities for interaction also within the classroom itself. This led to a more collaborative environment, more richly contextualized and continuously accessible that is the concept of ubiquitous and pervasive computing. The described case study could give a model for science learning in schools with a better interaction between the education and research environments.

Three classrooms from primary, secondary and high schools ${ }^{3}$ have been selected to join in the project allowing also to test the approach to wireless technology and mobile devices on behalf of students of different ages. The learning train consists of three modules: in the first one students follow a course about an astrophysical concept in each classroom, then they attend a set of activities in an Astrophysical research institute to improve their knowledge interacting with astronomers, and later on they done a final summary module.

${ }^{2}$ TabletPC is provided with Microsoft Windows XP Tablet PC edition [6] that is a Windows XP Professional enhanced version to exploit the features of this special notebook, while iPAQ uses Microsoft Windows Mobile 2003 for Pocket PC [7] that is a less complex operating system as that used by personal computer.

${ }^{3}$ A primary school ("Scuola Elementare Tempesta") a secondary school ("Scuola Media Peopoli") of Bologna (Italy) and one high school ("Liceo Scientifico Fracastoro") of Verona (Italy) joined in the project. Children were respectively 8,13 and 18 years old. 


\section{THE CASE STUDY: A WIRELESS NETWORK INFRASTRUCTURE FOR AN "EXTENDED" ELECTRONIC CLASSROOM}

The case study refers to the realization of an environment for learning and teaching activity such a classroom that is extended independently by the physical location: the idea is to apply wireless technology to build a highly interactive and mobile learning system using the mobility and portability features of the devices to allow ubiquitous learning. Students should use mobile devices to learn individually whenever they are and where they want (in classroom or in laboratory at school, at home or in another place such as different rooms of a research environment). They could interact each other in order to realize group work in and outside the school and to refer with the teacher/astronomer providing the didactic content, verifying and monitoring the learning phases. The activities to be deployed in several locations consist for example in reading digitized contents, practicing assignment while teacher assist them with multimedia context such as presentation slides, web pages, videos, online quizzes, e.g.

\subsection{Design of the system architecture}

According to the requirements ${ }^{4}$, each student has two handled devices - a tabletPC and a iPAQ - to participate in learning activities for individual and group-based competition. These devices should interact each others to exchange messages and materials for the group work and in the same time they should be connected to a main computer that acts as a server sharing and providing contents as the figure 1 shows. The device becomes the virtual interactive classroom server (equipped with the Apache HTTP [9] web server) for the activities of keeping records of individual student's learning portfolio and supporting teacher to give feedback to students. Moreover the same server could provide print and monitor functionalities sharing a printer and a digital projector. This interactive system requires the implementation of a wireless LAN (WLAN) [10] to provide locationindependent network access over radio waves to all resources and services useful for learning. The connection to Internet through a wired network

${ }^{4}$ Learning activities are designed by teachers and astronomers involved in the project: in particular the didactic design and its realization in each classroom has been carried out by Angela Turricchia of the "Planetario - Settore Istruzione - Comune di Bologna" and Maria Antonietta Carrozza of the "Liceo Fracastoro" School of Verona, while in the research institute by astronomers Caterina Boccato, Luca Nobili and Elena Lazzaretto of INAF. 
could increase learning train including search for information as one of the teaching activities.

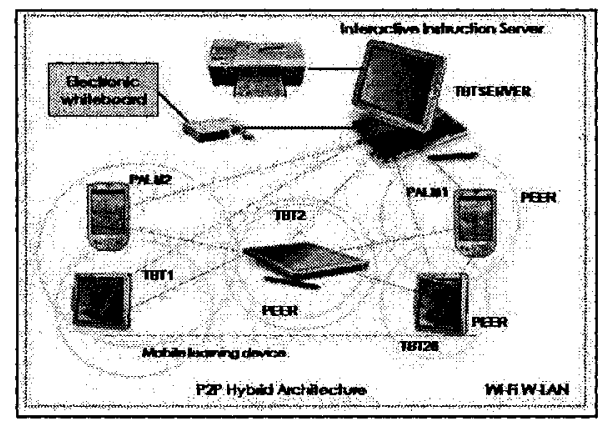

Figure 1. [Network infrastructure for the interactive system: the virtual classroom]

\subsection{Technological solutions for wireless network topologies and standards}

Basically speaking the effective range or distance reached by the network determines the type of network or standards. Each handled device could use the common wireless technologies: infrared, Bluetooth and Wi-Fi, the friendlier name for the IEEE 802.11b standard. The use of infrared port has been soon abandoned since the requirement of exchanging multimedia context in a fast way and between more devices. Bluetooth and Wi-Fi standards are both used for WLAN deployment suitable for the learning information system since they realize the two topology modes: ad hoc/ MANET [11] or infrastructure mode. The two topologies address different scenarios and system architectures. As regards wireless connectivity, the key difference between the Bluetooth and Wi-Fi standards is the expected operational rate: while operating in the same spectrum and suffering from the same level of interference Bluetooth is a short-range network that offers lower speed (2Mbps), while Wi-Fi is designed to offer full LAN connectivity and support the suite of networking protocols (i.e. TCP/IP) with theoretical $11 \mathrm{Mbps}$ of data rate. Moreover Bluetooth technology has a limit in the maximum number of connected devices without an appropriate access point. For these considerations Wi-Fi standard has been chosen to implement the WLAN for learning environment in both the two network topology modes allowing the use of such information system in different physical locations (figure 2). 


\subsubsection{Wireless ad hoc network topology}

The MANET consists of mostly homogeneous wireless links based on standard medium access control (MAC) where mobile nodes dynamically form a temporary network without the use of any existing wired network infrastructure or centralized administration and communicate directly with one another in a mesh to mesh topology (sometimes referred as Independent Basic Service Set - IBSS - topology). Owing to the limited radio propagation range of the wireless devices used, messages among nonneighbor nodes go through multiple intermediate nodes to reach destinations. Because of the multi-hop communication even for short geographical distances and random movement of mobile nodes, applications of ad hoc wireless networks are mainly restricted to small wireless islands which can be useful for intranet applications. Windows XP operating system supports the enabling of wireless of ad-hoc network both for Wi-Fi and Bluetooth technologies. The "Windows Connect Now" technology embedded in Windows XP Service Pack 2 (SP2) allows for creating a wireless network able to connect each network adapter. A pure ad hoc network using Wi-Fi wireless standard has been implemented for the classroom work: a small intranet has been manually created for each of the three classroom with a specific SSID. Within the network it could be possible to enable the ability to share files and directory for each nodes becoming part of a well-defined workgroup. All the devices must have an unique name to be identified and exchange information. The security problem in this deployment, has not be taken in consideration due to the locality of the network in a classroom context. This infrastructure gives the opportunity to exchange contents of all formats and to provide web access to resources: using the Windows network functionalities the tablets' network work well and it is possible to identify each one in the network. Problems arise with iPAQs since their operating system doesn't permit to assign an IP and every device is shown with the generic name "Pocket PC" making impossible their network unique identification. Moreover, the ad-hoc network implementation shown some connectivity issues when all the devices are connected to the wireless network, and obviously performance problems in the simultaneous exchange of multimedia content due to the limited band.

\subsubsection{Infrastructure topology: W-LAN with access point}

Generally using the infrastructure mode of 802.11 standard (known as Basic Service Set - BSS), devices requires the installation of at least one Access Point (AP) referred to as a base station connected to the wired network. In this context all the communications also those between the 
nodes occur via the AP. Wireless nodes have to establish a relation of association before the messages exchange: they listen for beacon messages to identify the access points within the range. However if such wireless network are badly designed W-LAN access points can become bottlenecks. In this context an AP could improve data transmission, since all available devices support the $802.11 \mathrm{~g}$ standard enabling a rate of $54 \mathrm{Mbs}$. This configuration could not be adopted in the school due to the lack of a wired network infrastructure, but only in the research institute where students attend a course's module structured in several activities.

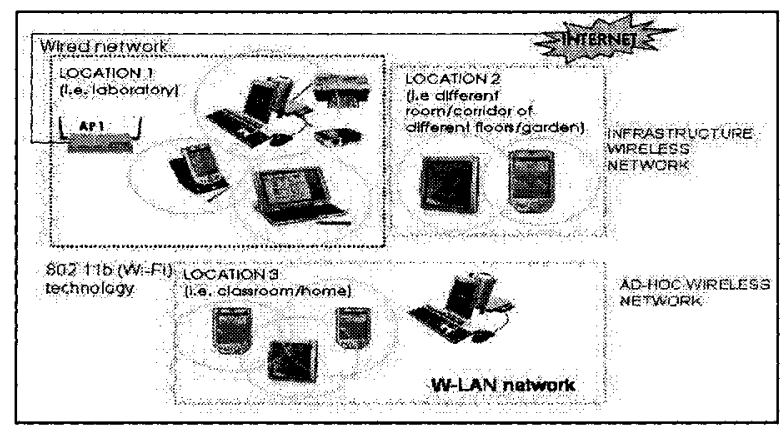

Figure 2. [W-LANs for several physical location in intranet and internet context]

In this location, the existent wireless network is expanded adding multiple APs which are combined into a single sub-network (as in the Extended Service Set - ESS) topology. It has been decided to use the existent WLAN infrastructure, also because since the Wi-Fi standard does not define roaming techniques, there could be problems when roaming users crossed a router boundary between subnets. Adopting DHCP, it is necessary to force users to release IP address as migrate from one subnet to another. A detail plan for installation of these devices is necessary for interference problems, since wireless network and any data transmitted over it employ radio signals and broadcast that extend in all direction. The overlapping problem is solved using different radio channels to boost aggregate throughput and this change has really improve performance issues. Finally as regards security and the three main methods [12] built into 802.11 networks (SSID, MAC address filtering and WEP), the MAC address filtering has been chosen both for the fact that the devices are known at start point and the necessity to don't introduce complexity in the management of the whole network. With this configuration, the wireless network work well and with good performance for all the learning activities done in different rooms of 
the institute and also outside it (in the garden): this test contribute to really show students and teachers the efficacy of the mobility feature.

\subsection{Encountered Issues}

The main issues encountered in the network deployment are the following:

- iPAQs problems with inter-connectivity and limited software

- unexpected lost of signal in ad-hoc network topology

- limited band of $802.11 \mathrm{~b}$ protocol in exchanging simultaneous multimedia context both in ad-hoc and infrastructure mode.

The issue of the iPAQs network unique identification that limits the possibility to control them and to interact directly with tablets, is probably due to the software. A test using tablet's Bluetooth feature and its functionalities (figure 3) permits to assign a unique name for each iPAQ with the paring option, but this connection don't give full control of each student's PDA and could be used only in a limited context such as at home for example.

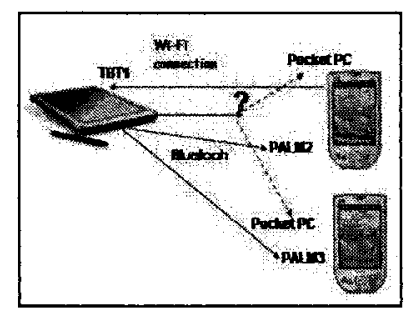

Figure 3. [Issues for recognizing palm in unique way with Wi-Fi connectivity]

Other software problems using devices regard the network file transmission that could not be done using the basic functionality of the IPAQ operating system. Students use normally the "drag \& drop" utility also for transferring files and it is difficult for them to follow a set of steps to transfer a single file, such as using the basic procedure. The issue is partially solved by the use of an $\mathrm{ftp}$ server installed on the interactive server, but it's on study the way to realize an effective interaction between all these devices. Microsoft Mobile software has the possibility to synchronize remotely the IPAQ with a server using the ActiveSync software, but this option is available only for a limit set of applications (Calendar, Contact or Inbox). The goal is to develop an application able to provide this functionality. 


\section{CONCLUSIONS}

WLAN offers flexibility but the factors implementing a successful wireless network are: user interface, network logon rate, response time, throughput and reliable data delivery. The first test of this W-LAN deployment has demonstrate its effectiveness both in each school than in the research institute environment. The wireless network is up to now widely use in each classroom for learning and teaching activities due also for the easy way of set up and use even if some issues remain regarding performance and a sometime unexpected lost of connectivity. On the other hand the WLAN implemented in the wired infrastructure has permitted to make all the learning/teaching programmed activities with a reliable data delivery and a good response time. The learning modules developed using all formats (e.g video and animation) could permit a really test of the network that had maintained a good performance. To continue the experiment with other schools and make a more direct link between education and research world, it will be necessary to design two different WLAN networks in order to separate the student network by that of researcher and also really face security aspects.

\section{ACKNOLEDGMENTS}

The author likes to acknowledge HP Philanthropy, the project head Prof. Leopoldo Benacchio, the astronomers Caterina Boccato, Luca Nobili, Elena Lazzaretto of INAF, Angela Turricchia of the "Planetario - Settore Istruzione - Comune of Bologna and Maria Antonietta Carrozza of the Fracastoro School of Verona involved in the project and Roberto Greggio for his help in the testing phase.

\section{REFERENCES}

[1] Mark Weiser, "The Computer for the Twenty-First Century," Scientific American, pp. 9410 , September 1991

[2] HP Philanthropy, http://grants.hp.com/

[3] HP Tablet PC TC1100 http:/h18000.www1.hp.com/products/tabletpc/tc1 100/

[4] IEEE Standards Department. IEEE 802.11 standard for wireless LAN, medium access control (MAC) and physical layer (PHY) specifications, 1997

[5] IEEE Standards Department. IEEE Bluetooth standard: Wireless mac and phy specifications for wireless personal area networks (wpans[tm]), 1997

[6] Windows $X P \quad$ Tablet PC Home Page, https://s.microsoft.com/windowsxp/tabletpc/default.mspx 
[7] Windows Mobile 2003 for Pocket PC, http://www.microsoft.com/windowsmobile/pocketpc/ppc/default.mspx

[8] Mobile Learning and Pervasive Computing, http://www3.telus.net/ kdeanna/mlearning/

[9] The Apache HTTP Server, http://www.apache.org

[10] Wireless LAN (WLAN), http://www.ukoln.ac.uk/public/earl/issuepapers/wireless.html

[11] Mobile Ad-hoc Networks (manet), http://www.ietf.org/html.charters/manet-charter.html

[12] WLAN security measures, http://www.backupbook.com/02Security/A1WLAN_Security.html 